\title{
EI PANORAMA DE LAS INVESTIGACIONES SOBRE FINANZAS SOCIALES \\ THE OVERVIEW OF SOCIAL FINANCE RESEARCH
}

\author{
Juan Carlos Lezama Palomino
}

DOI: 10.13140/RG.2.2.28318.15684

\section{Resumen}

En los últimos años ha surgido una corriente en las finanzas con fines sociales, aunque el concepto de combinar los objetivos sociales y financieros no es nuevo, después de la crisis del 2008 se empieza a tomar mayor fuerza y reconocimiento mundial. Las finanzas sociales surgen como una respuesta al uso indebido de las finanzas, así como los inversores confían en los métodos de inversión que ha servido en los negocios, estas mismas técnicas se podría aplicar para solucionar problemas sociales, reduciendo brechas y apoyando al logro de los ODS. Al ser una corriente nueva el presente trabajo busca revisar el estado de las investigaciones de los últimos 10 años a través de las bases de datos de Scopus, Scimago y LaReferencia.

\begin{abstract}
In recent years, a trend in finance with social goals has emerged, although the concept of combining social and financial goals is not new, after the 2008 crisis it has gained greater strength and global recognition. Social finance has emerged as a response to the abuse of finance, just as investors have confidence in the investment methods that have served business, these same techniques could be applied to solve social problems, reducing gaps and helping to realize Sustainable Development Goals. Being a new trend, this paper reviews the state of research of the last 10 years through the databases of Scopus, Scimago and LaReferencia.
\end{abstract}

Palabras claves: finanzas sociales, Objetivos del Desarrollo Sostenible, Inversión social

Key words: social finance, Sustainable Development Goals, social investment 


\section{EI PANORAMA DE LAS INVESTIGACIONES SOBRE FINANZAS SOCIALES}

\section{Contexto Internacional:}

Las finanzas sociales es una corriente alterna de las finanzas que va más allá de la perspectiva tradicional de buscar una mayor rentabilidad y utilidad a lograr unos objetivos sociales. Esta corriente toma fuerza en el mundo a partir de la crisis económica del 2008, pero no se ha identificado el país que procede pues las temáticas se han abordado en diferentes regiones, principalmente en Europa. Sin embargo, la investigación en las finanzas las lidera a nivel mundial Estados Unidos, seguido de Reino Unido, China y Alemania. (Ver Tabla 1).

Tabla 1 Ranking globlal en investigación en finanzas desde 1996 al 2018

\begin{tabular}{|c|c|c|c|c|c|c|c|c|}
\hline & Coun & & $\downarrow$ Documents & Citable documents & Citations & Self-Citations & Citations per Document & Hindex \\
\hline 1 & 需 & United States & 58347 & 55741 & 1561614 & 648301 & 26.76 & 413 \\
\hline 2 & 背 & United Kingdom & 17356 & 16675 & 313966 & 67975 & 18.09 & 185 \\
\hline 3 & 2 & China & 8826 & 8494 & 58259 & 20950 & 6.60 & 88 \\
\hline 4 & $=$ & Germany & 8595 & 8350 & 110894 & 24141 & 12.90 & 115 \\
\hline 5 & 용 & Australia & 7844 & 7497 & 100331 & 20558 & 12.79 & 115 \\
\hline 6 & $|+|$ & Canada & 6842 & 6562 & 122121 & 14799 & 17.85 & 136 \\
\hline 7 & 11 & France & 6014 & 5755 & 82557 & 12603 & 13.73 & 108 \\
\hline 8 & $=$ & Spain & 4302 & 4190 & 60083 & 11302 & 13.97 & 101 \\
\hline 9 & 11 & Italy & 4236 & 4097 & 61379 & 11490 & 14.49 & 102 \\
\hline 10 & $=$ & Netherlands & 3774 & 3637 & 83759 & 8626 & 22.19 & 120 \\
\hline
\end{tabular}

Fuente: Tomado de Scimago Journal \& Country Rank (2020)

Aunque Colombia no lidera el ranking, se encuentra en el puesto 48 a nivel mundial de 180 países estudiados (Scimago Journal \& Country Rank, 2020). Además, ocupa el 4 lugar de los países latinoamericanos siendo el primero Brasil (Ver Tabla 2). 
Tabla 2 Ranking latinoamericano en investigación en finanzas desde 1996 al 2018

\begin{tabular}{|c|c|c|c|c|c|c|c|}
\hline & Country & $\downarrow$ Documents & Citable documents & Citations & Self-Citations & Citations per Document & Hindex \\
\hline & Brazil & 874 & 839 & 5868 & 1014 & 6.71 & 34 \\
\hline 2 & L Chile & 509 & 478 & 6768 & 441 & 13.30 & 34 \\
\hline 3 & I. Mexico & 352 & 333 & 3331 & 247 & 9.46 & 32 \\
\hline 4 & Colombia & 251 & 239 & 1177 & 153 & 4.69 & 16 \\
\hline 5 & I Argentina & 241 & 227 & 4196 & 190 & 17.41 & 33 \\
\hline 6 & I Peru & 66 & 62 & 426 & 21 & 6.45 & 10 \\
\hline 7 & $\stackrel{\equiv \text { Uruguay }}{=}$ & 47 & 44 & 381 & 27 & 8.11 & 10 \\
\hline 8 & - Venezuela & 31 & 31 & 282 & 20 & 9.10 & 9 \\
\hline 9 & X Jamaica & 30 & 29 & 165 & 4 & 5.50 & 6 \\
\hline & $E$ Puerto Rico & 28 & 28 & 92 & 11 & 3.29 & 5 \\
\hline
\end{tabular}

Fuente: Tomado de Scimago Journal \& Country Rank (2020)

Con respecto a las publicaciones más importantes a nivel mundial se encuentra en primer lugar la revista Journal of Finance de Reino Unido, Journal of Financial Economics de Países Bajos y Review of Financial Studies de Reino Unido. Como se puede observar en la Tabla 3 las revistas que lideran los primeros puestos del ranking son de Reino Unido, Países Bajos y Estados Unidos.

Al analizar la situación de publicaciones en América Latina se encuentra solamente tres revistas, la cual ocupa el primer lugar la Revista Contabilidade e Financas de Brasil, seguido de Economía Chilena de Chile y Revista Finanzas y Política Económica de Colombia. 
Tabla 3 Ranking global de las publicaciones de journals al corte de 2018

\begin{tabular}{|c|c|c|c|c|c|c|c|c|c|c|c|c|}
\hline & Title & Type & $\downarrow S J R$ & $\begin{aligned} H \\
\text { index }\end{aligned}$ & $\begin{array}{c}\text { Total } \\
\text { Docs. } \\
(2018)\end{array}$ & $\begin{array}{l}\text { Total Docs. } \\
\text { (3years) }\end{array}$ & $\begin{array}{l}\text { Total Refs. } \\
\text { (2018) }\end{array}$ & $\begin{array}{r}\text { Total Cites } \\
\text { (3years) }\end{array}$ & $\begin{array}{l}\text { Citable Docs. } \\
\text { (3years) }\end{array}$ & $\begin{array}{l}\text { Cites / Doc. } \\
\text { (2years) }\end{array}$ & $\begin{array}{l}\text { Ref./ Doc. } \\
\quad(2018)\end{array}$ & \\
\hline 1 & Journal of Finance & journal & $\begin{array}{r}17.973 \\
\text { Q1 }\end{array}$ & 264 & 67 & 216 & 3606 & 1617 & 209 & 6.28 & 53.82 & 慗 \\
\hline 2 & Journal of Financial Economics & journal & $\begin{array}{c}13.636 \\
\text { Q1 }\end{array}$ & 223 & 128 & 368 & 6824 & 2702 & 362 & 5.04 & 53.31 & $=$ \\
\hline 3 & Review of Financial Studies & journal & $\begin{array}{r}12.516 \\
\text { Q1 }\end{array}$ & 157 & 124 & 300 & 6634 & 1791 & 291 & 5.30 & 53.50 & 整 \\
\hline 4 & Journal of Accounting Research & journal & $\begin{array}{r}10.151 \\
\text { Q1 }\end{array}$ & 118 & 34 & 97 & 2090 & 599 & 91 & 5.52 & 61.47 & 米类 \\
\hline 5 & Journal of Management & journal & $\begin{array}{r}7.936 \\
\text { Q1 }\end{array}$ & 192 & 167 & 244 & 13856 & 2680 & 220 & 11.45 & 82.97 & 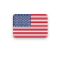 \\
\hline 6 & Journal of Monetary Economics & journal & $\begin{array}{r}7.248 \\
\text { Q1 }\end{array}$ & 112 & 79 & 216 & 2830 & 664 & 184 & 2.82 & 35.82 & $\equiv$ \\
\hline 7 & $\begin{array}{l}\text { Journal of Accounting and } \\
\text { Economics }\end{array}$ & journal & $\begin{array}{r}6.606 \\
\text { Q1 }\end{array}$ & 132 & 50 & 128 & 2647 & 613 & 127 & 3.89 & 52.94 & $=$ \\
\hline 8 & Accounting Review & journal & $\begin{array}{c}5.240 \\
Q 1\end{array}$ & 133 & 88 & 227 & 5260 & 1018 & 218 & 4.56 & 59.77 & 䨐 \\
\hline 9 & $\begin{array}{l}\text { Annual Review of Financial } \\
\text { Economics }\end{array}$ & journal & $\begin{array}{r}4.584 \\
\text { Q1 }\end{array}$ & 22 & 21 & 48 & 1875 & 161 & 48 & 2.58 & 89.29 & 㕵 \\
\hline 10 & Journal of International Economics & journal & $\begin{array}{r}4.347 \\
\text { Q1 }\end{array}$ & 121 & 92 & 278 & 4392 & 882 & 276 & 2.47 & 47.74 & $=$ \\
\hline
\end{tabular}

Fuente: Tomado de Scimago Journal \& Country Rank (2020)

Tabla 4 Ranking latinoamericano de las publicaciones de journals al corte de 2018

\begin{tabular}{|c|c|c|c|c|c|c|c|c|c|c|c|}
\hline & Title & Type & $\downarrow S J R$ & $\begin{array}{r}\mathrm{H} \\
\text { index }\end{array}$ & $\begin{array}{l}\text { Total Docs. } \\
\text { (2018) }\end{array}$ & $\begin{array}{l}\text { Total Docs. } \\
\text { (3years) }\end{array}$ & $\begin{array}{l}\text { Total Refs. } \\
\text { (2018) }\end{array}$ & $\begin{array}{r}\text { Total Cites } \\
\text { (3years) }\end{array}$ & $\begin{array}{l}\text { Citable Docs. } \\
\text { (3years) }\end{array}$ & $\begin{array}{l}\text { Cites / Doc. } \\
\text { (2years) }\end{array}$ & $\begin{array}{l}\text { Ref./ Doc. } \\
\quad(2018)\end{array}$ \\
\hline 1 & $\begin{array}{l}\text { Revista Contabilidade e } \\
\text { Financas } \precsim\end{array}$ & journal & $\begin{array}{c}0.179 \\
Q 4\end{array}$ & 3 & 31 & 95 & 1342 & 40 & 83 & 0.38 & 43.29 \\
\hline 2 & Economia Chilena & journal & $\begin{array}{l}0.161 \\
Q 4\end{array}$ & 4 & 5 & 47 & 142 & 6 & 40 & 0.12 & 28.40 \\
\hline 3 & $\begin{array}{l}\text { Revista Finanzas y Politica } \\
\text { Economica }\end{array}$ & journal & $\begin{array}{c}0.128 \\
04\end{array}$ & 2 & 18 & 18 & 672 & 4 & 16 & 0.25 & 37.33 \\
\hline
\end{tabular}

Fuente: Tomado de Scimago Journal \& Country Rank (2020)

Por otro lado, al analizar la ecuación de búsqueda ${ }^{1}$ sobre el tema de las finanzas sociales en Scopus, se encontró que el año con el mayor número de publicaciones relacionadas al tema es el año 2019 con 10573 (Ver Tabla 5). Llama la atención que el tema ha ido creciendo constantemente en los últimos años; el año 2020 hay una caída en las publicaciones, pero es porque el año no ha terminado.

\footnotetext{
1 Se utilizó la siguiente ecuación de búsqueda: (social finance) or (social financial) or ( social investment)
} 
Tabla 5 Documentos de finanzas sociales citados en los últimos 10 años Documents by year

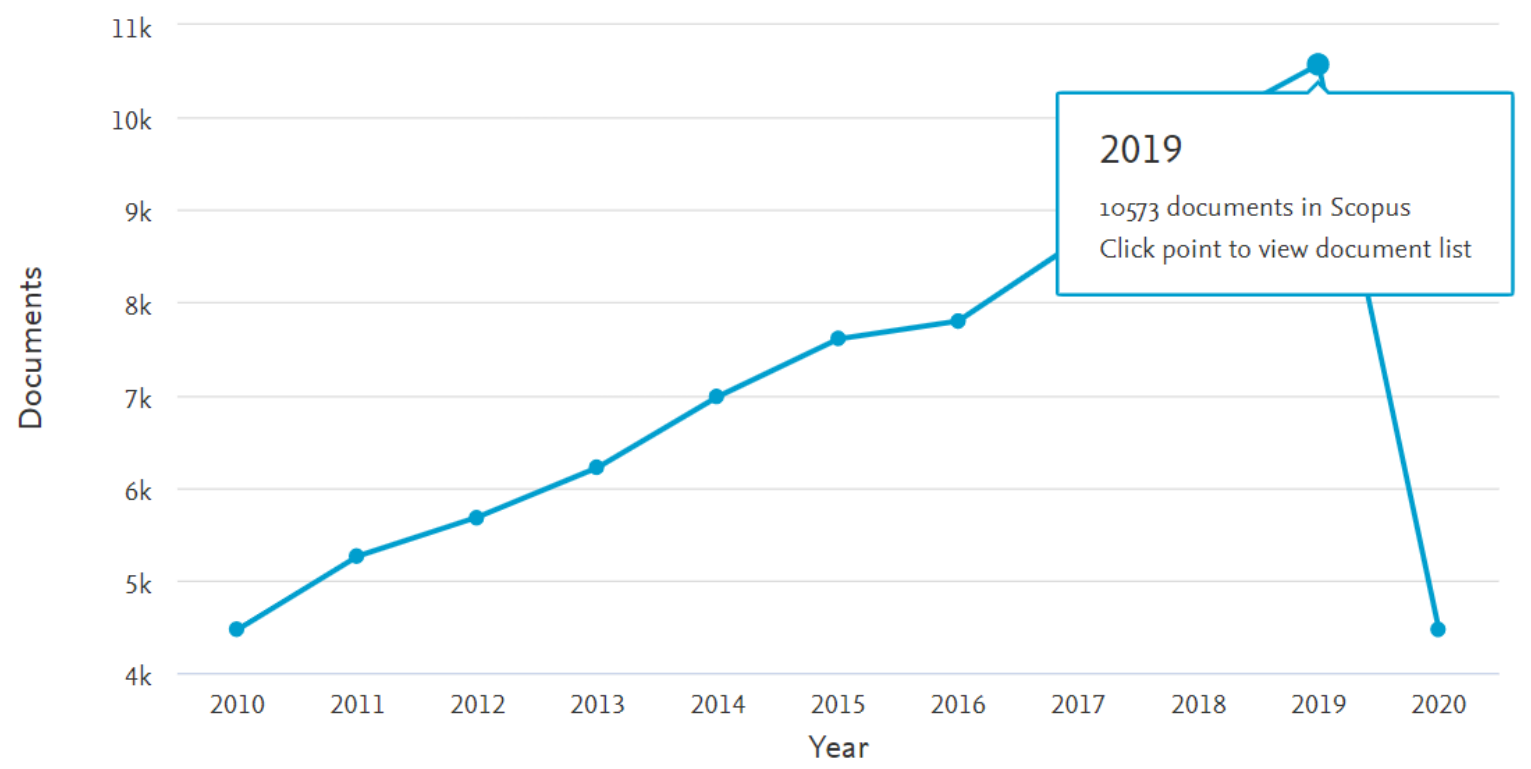

Fuente: Tomado de Scopus desde el 2010 al 2020 (Scopus, 2020)

Con respecto a los autores que más han realizado publicaciones, se encuentra en primer lugar McKee, M. con publicaciones en Reino Unido (42), Estados Unidos (19), Bélgica (6) y otros. El segundo autor con más citaciones es Stuckler, D. con publicaciones en Reino unido (29), Estados Unidos (16) e Italia (6), entre otros países (Scopus, 2020). En la Tabla 6 se relaciona los 10 autores principales con mayor producción en temas de finanzas sociales.

Tabla 6 Ranking de los autores con mayor producción en finanzas sociales citados en los últimos 10 años 


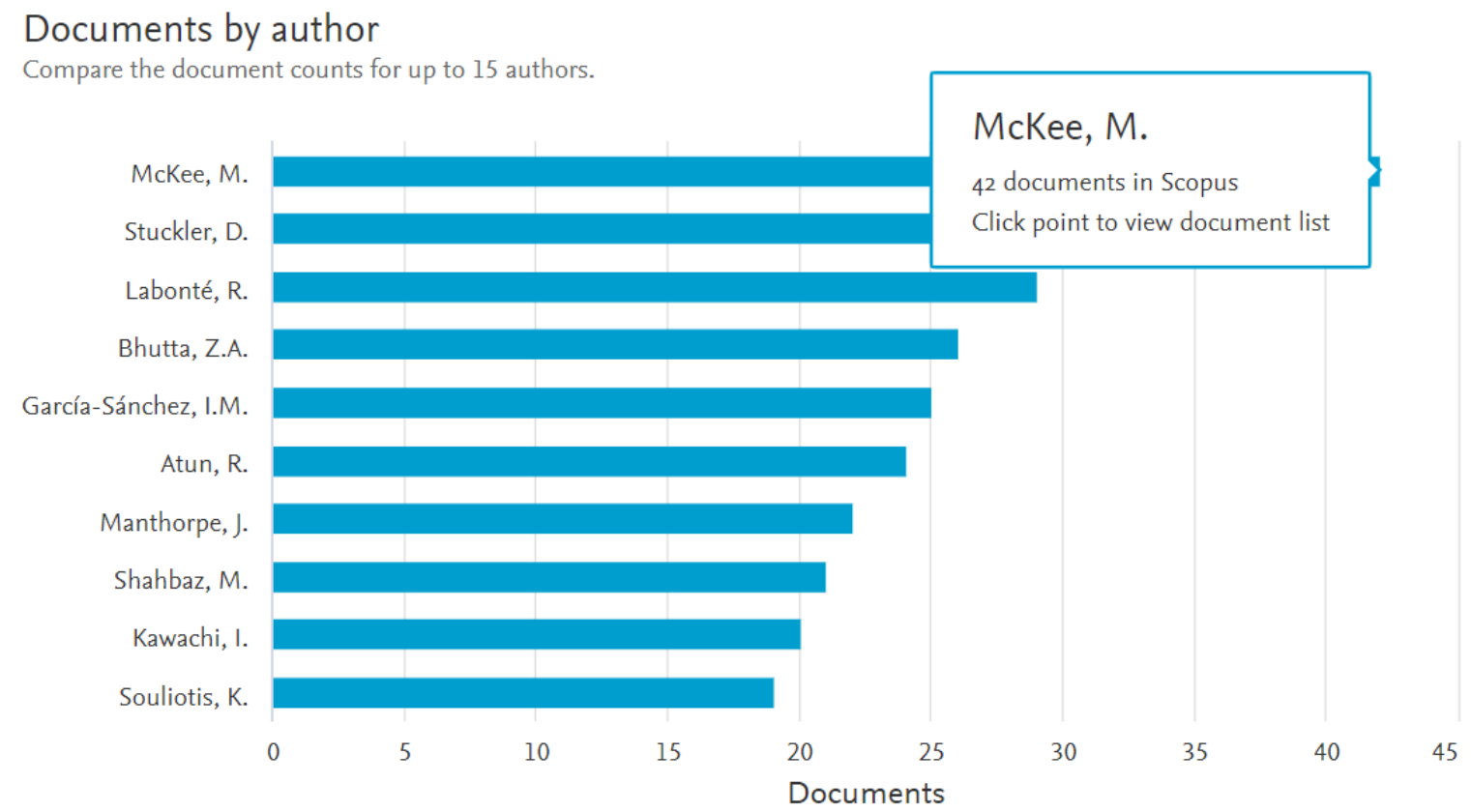

Fuente: Tomado de Scopus desde el 2010 al 2020 (Scopus, 2020)

En relación con las instituciones que más han apoyado las publicaciones, como se puede apreciar en la Tabla 6, se encuentra la Universidad de Oxford con 645 documentos, seguido de University College de Londres con 592 publicaciones, ambas de Reino Unido, y en tercer lugar la Universidad de Toronto con 573 publicaciones.

Tabla 7 Ranking de las instituciones con mayor producción en finanzas sociales citados en los últimos 10 años 
Documents by affiliation

Compare the document counts for up to 15 affiliations.

University of Oxford

$$
\text { UCL }
$$

University of Toronto

University of Melbourne

The University of Sydney

University of New South Wales UNSW A...

The University of Queensland

King's College London

London School of Hygiene \&amp; Tropi...

University of Cambridge

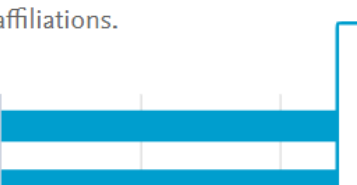

University of Oxford

645 documents in Scopus

Click chart segment to view document list

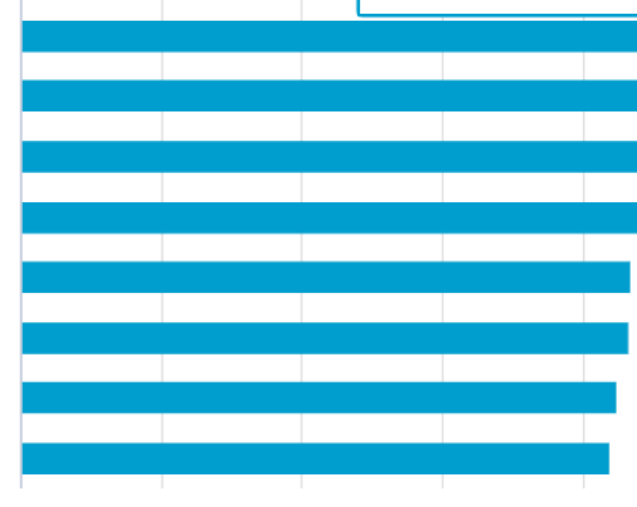

$100 \quad 200$

300

400

Documents

Fuente: Tomado de Scopus desde el 2010 al 2020 (Scopus, 2020)

Por otra parte, el $70,7 \%$ de las publicaciones relacionadas con el tema de finanzas sociales son artículos, seguido de un $10,8 \%$ de publicaciones de conferencias y un $6,4 \%$ son reseñas (Ver tabla 8 ). 
Tabla 8 Tipo de producción en temas de finanzas sociales citados en los últimos 10 años

Documents by type

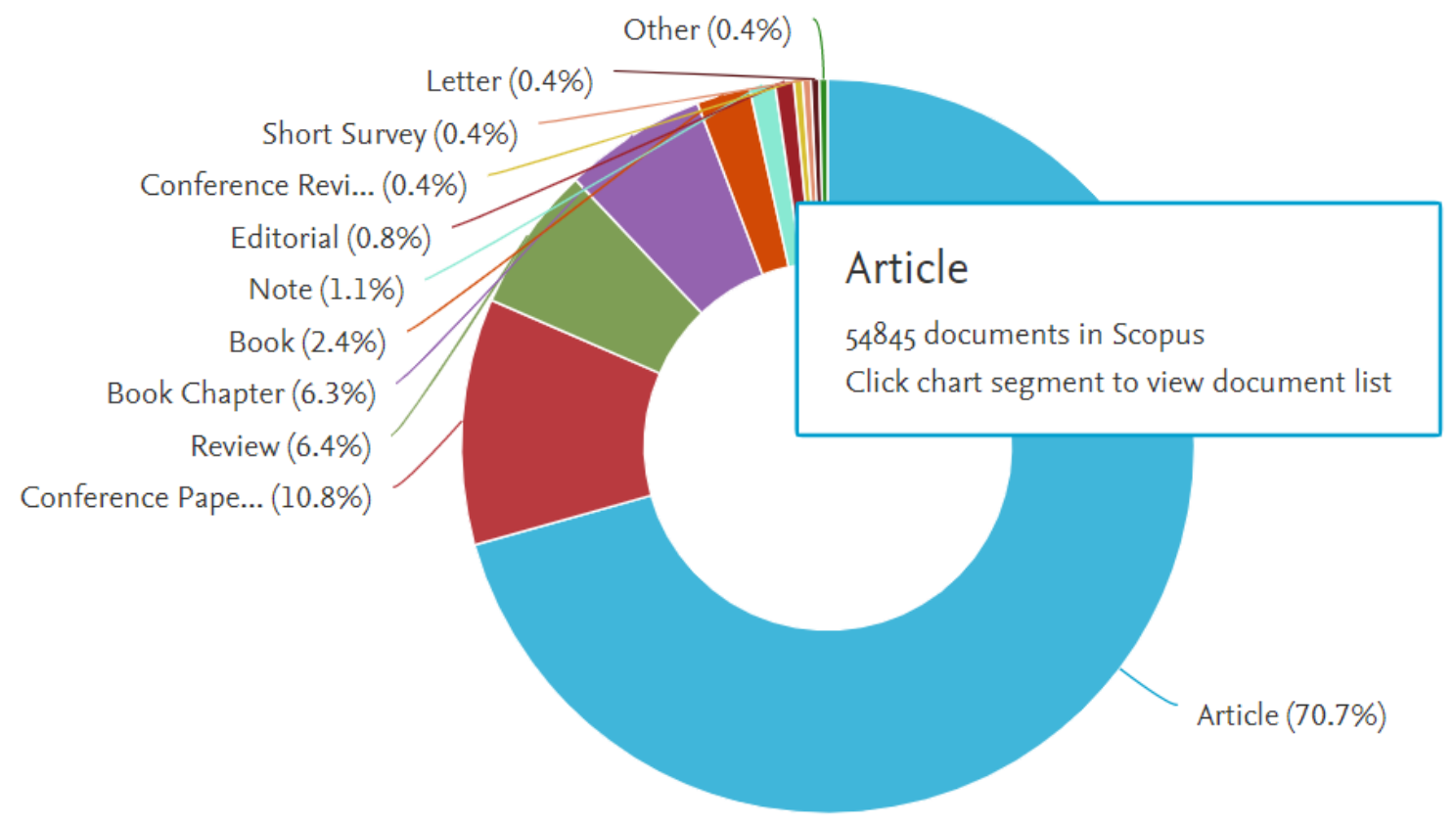

Fuente: Tomado de Scopus desde el 2010 al 2020 (Scopus, 2020)

\section{Contexto regional}

Con respecto a la producción latinoamericana, de acuerdo con la La Referencia (2020), Colombia es el primer país de la región con el mayor número de publicaciones relacionadas con el tema ${ }^{2}$; seguido de Perú, Ecuador y Costa Rica (ver tabla 9).

Tabla 9 Tipo de producción en temas de finanzas sociales citados en Latinoamérica

2 La ecuación de búsqueda utilizada fue: (finanzas sociales) or (inversión social) 


\begin{tabular}{|lr|}
\hline País & \multicolumn{1}{|c|}{} \\
\hline Colombia & 13 \\
\hline Perú & 9 \\
\hline Ecuador & 2 \\
\hline Costa Rica & 1 \\
\hline
\end{tabular}

Fuente: Tomado de LA Referencia (2020)

Con relación a los autores que más escriben del tema, no es concluyente pues la mayoría solo tiene una sola publicación, por lo cual no hay algún autor que se destaque en los países latinoamericanos.

Tabla 10 Autores con producción en temas de finanzas sociales en Latinoamérica

\begin{tabular}{|lr|}
\hline \multicolumn{1}{|c|}{ Autor } \\
\hline Magíster en Gerencia Estratégica & 2 \\
\hline Arango Escobar, Valentina & 1 \\
\hline Arrarte Mera, Raúl Alberto & 1 \\
\hline Borda López, Jorge Andrés & 1 \\
\hline Borja Rueda, Carlos Alberto & 1 \\
\hline Cabrera Lavado, Julio César & 1 \\
\hline Cabrera Tobar, Verónica Noemí & 1 \\
\hline Calle Forero, Jorge Alberto & 1 \\
\hline Campbell Falcón, Carmen Brigitte & 1 \\
\hline De la Torre Cortéz, Dennys Dunker & 1 \\
\hline
\end{tabular}

Fuente: Tomado de LA Referencia (2020)

Por otra parte, las instituciones con mayor producción en temas de finanzas sociales en Latinoamérica son de Colombia, aunque la que ocupa el primer lugar es de Perú 
con 5 publicaciones. La cantidad de publicaciones en nuestros países es baja en comparación a las que se realizan en el mundo.

Tabla 11 Instituciones con producción en temas de finanzas sociales en Latinoamérica

\begin{tabular}{|l|}
\hline 命 Institución \\
\hline $\begin{array}{l}\text { Pontificia Universidad Católica del } \\
\text { Perú }\end{array}$ \\
\hline Universidad del Rosario \\
\hline Universidad Nacional de Colombia \\
\hline Universidad de la Sabana \\
\hline Universidad Militar Nueva Granada \\
\hline Universidad Nacional de Ingeniería \\
\hline Universidad Técnica de Ambato \\
\hline Universidad Nacional Mayor de San \\
\hline Marcos
\end{tabular}

Fuente: Tomado de LA Referencia (2020)

Por último, la mayoría de las publicaciones son tesis de maestría con 22, seguido de 2 artículos y una tesis doctoral (Ver Tabla 12), en comparación a lo que sucede en el mundo, la mayoría de las publicaciones son artículos.

Tabla 12 Tipo de producción en temas de finanzas sociales en Latinoamérica

\begin{tabular}{|lr|}
\hline 品I Tipo de recurso & \multicolumn{1}{|c|}{} \\
\hline tesis de maestría & 22 \\
\hline artículo & 2 \\
\hline tesis doctoral & 1 \\
\hline
\end{tabular}


Fuente: Tomado de LA Referencia (2020)

\section{Estudio cienciométrico}

A continuación, se hace un resumen cienciometrico de las investigaciones relacionadas con el tema de finanzas sociales:

Tabla 13 Producción en temas de finanzas sociales

\begin{tabular}{|c|c|c|c|c|c|}
\hline Indicador & Descripción & Fuente & 2018 & 2019 & 2020 \\
\hline $\begin{array}{l}\text { Indicador de } \\
\text { producción }\end{array}$ & Total de artículos & Scopus & 6828 & 8037 & 3741 \\
\hline $\begin{array}{l}\text { Indicador } \quad \text { de } \\
\text { producción }\end{array}$ & Total de libros & Scopus & 262 & 181 & 71 \\
\hline $\begin{array}{l}\text { Indicador de } \\
\text { producción }\end{array}$ & Total de capítulos de libros & Scopus & 652 & 322 & 101 \\
\hline $\begin{array}{l}\text { Indicador de } \\
\text { producción }\end{array}$ & Total de Conference Paper & Scopus & 1168 & 1277 & 270 \\
\hline $\begin{array}{l}\text { Indicador } \quad \text { de } \\
\text { producción }\end{array}$ & Principal entidad financiadoras & Scopus & 99 & 80 & 33 \\
\hline $\begin{array}{l}\text { Indicador de } \\
\text { producción }\end{array}$ & $\begin{array}{l}\text { Principal país de publicación (sin incluir el país de } \\
\text { origen) }\end{array}$ & Scopus & 2345 & 2371 & 1013 \\
\hline $\begin{array}{l}\text { Indicador de } \\
\text { producción }\end{array}$ & Autor con mayor publicaciones & Scopus & 3 & 1 & 1 \\
\hline $\begin{array}{l}\text { Indicador } \quad \text { de } \\
\text { producción }\end{array}$ & Total de artículos & wOS & 6570 & 7531 & 2746 \\
\hline $\begin{array}{l}\text { Indicador } \quad \text { de } \\
\text { producción }\end{array}$ & Total de libros & WOS & 16 & 32 & 4 \\
\hline $\begin{array}{l}\text { Indicador de } \\
\text { producción }\end{array}$ & Total de capítulos de libros & WOS & 18 & 7 & 1 \\
\hline $\begin{array}{l}\text { Indicador } \quad \text { de } \\
\text { producción }\end{array}$ & Total de Conference Paper & wOS & 8 & 7 & 1 \\
\hline $\begin{array}{l}\text { Indicador de } \\
\text { producción }\end{array}$ & Principal entidad financiadoras & WOS & 180 & 233 & 787 \\
\hline $\begin{array}{l}\text { Indicador de } \\
\text { producción }\end{array}$ & $\begin{array}{l}\text { Principal país de publicación (sin incluir el país de } \\
\text { origen) }\end{array}$ & WOS & 1697 & 1758 & 719 \\
\hline $\begin{array}{l}\text { Indicador de } \\
\text { producción }\end{array}$ & Autor con mayor publicaciones & wOS & 10 & 8 & 5 \\
\hline $\begin{array}{l}\text { Indicador } \quad \text { de } \\
\text { producción }\end{array}$ & Total de artículos & LaReferencia & 0 & 0 & 1 \\
\hline $\begin{array}{l}\text { Indicador de } \\
\text { producción }\end{array}$ & Total de libros & LaReferencia & 0 & 0 & 0 \\
\hline $\begin{array}{l}\text { Indicador } \quad \text { de } \\
\text { producción }\end{array}$ & Total Tesis Doctorado & LaReferencia & 0 & 1 & 0 \\
\hline $\begin{array}{l}\text { Indicador } \quad \text { de } \\
\text { producción }\end{array}$ & Total de Maestría & LaReferencia & 7 & 2 & 0 \\
\hline $\begin{array}{l}\text { Indicador de } \\
\text { producción }\end{array}$ & $\begin{array}{l}\text { Principal país de publicación (sin incluir el país de } \\
\text { origen) }\end{array}$ & LaReferencia & 5 & 0 & 0 \\
\hline $\begin{array}{l}\text { Indicador de } \\
\text { producción }\end{array}$ & Autor con mayor publicaciones & LaReferencia & 2 & 0 & 0 \\
\hline $\begin{array}{l}\text { Indicador de } \\
\text { producción }\end{array}$ & Total de artículos & Scielo & 234 & NA & NA \\
\hline $\begin{array}{l}\text { Indicador } \quad \text { de } \\
\text { producción }\end{array}$ & Principal entidad financiadoras & Scielo & NA & NA & NA \\
\hline $\begin{array}{l}\text { Indicador de } \\
\text { producción }\end{array}$ & $\begin{array}{l}\text { Principal país de publicación (sin incluir el país de } \\
\text { origen) }\end{array}$ & Scielo & 82 & 0 & 0 \\
\hline $\begin{array}{l}\text { Indicador } \quad \text { de } \\
\text { producción }\end{array}$ & Autor con mayor publicaciones & Scielo & NA & NA & NA \\
\hline $\begin{array}{l}\text { Indicador } \quad \text { de } \\
\text { producción }\end{array}$ & Total de publicaciones & Google Scholar & 157000 & 103000 & 73300 \\
\hline
\end{tabular}

Fuente Elaboración Propia 
A continuación, se muestra los siguientes indicadores más importantes de las publicaciones relacionadas con las finanzas sociales comparadas entre Colombia y el mundo

Tabla 14 Indicadores de Producción en temas de finanzas sociales ${ }^{3}$

\begin{tabular}{|c|c|c|c|c|c|}
\hline Indicador & Descripción & Fuente & 2018 & 2019 & 2020 \\
\hline Índice de actividad & $\begin{array}{llll}\begin{array}{l}\text { tema, área de } \\
\text { disciplina }\end{array} & \text { conocimiento } \mathrm{y} / \mathrm{o} \\
\end{array}$ & Scopus & $0.67 \%$ & $0.52 \%$ & $0.47 \%$ \\
\hline Índice de actividad & $\begin{array}{l}\text { tema, área de conocimiento } \\
\text { disciplina }\end{array}$ & wOS & $0.76 \%$ & $0.66 \%$ & $0.85 \%$ \\
\hline $\begin{array}{l}\text { Tasa de } \\
\text { crecimiento }\end{array}$ & $\begin{array}{l}\text { tema, área de conocimiento } \mathrm{y} / \mathrm{o} \\
\text { disciplina }\end{array}$ & Scopus & $\begin{array}{l}\text { Mundo: } \\
13.8 \% \\
\text { Col: } 34.7 \%\end{array}$ & $\begin{array}{l}\text { Mundo: } 7.1 \% \\
\text { Col: }-16.7 \%\end{array}$ & \begin{tabular}{|l|} 
Mundo: \\
$57.7 \%$ \\
Col: $-61.8 \%$ \\
\end{tabular} \\
\hline $\begin{array}{l}\text { Tasa de } \\
\text { crecimiento }\end{array}$ & $\begin{array}{l}\text { tema, área de conocimiento y/o } \\
\text { disciplina }\end{array}$ & wos & $\begin{array}{l}\text { Mundo: } \\
18.6 \% \\
\text { Col: } 17.4 \%\end{array}$ & \begin{tabular}{|l|} 
Mundo: \\
$13.3 \%$ \\
Col: $-1.9 \%$ \\
\end{tabular} & \begin{tabular}{|l|} 
Mundo: \\
$63.2 \%$ \\
Col: $-52.8 \%$ \\
\end{tabular} \\
\hline Índice de impacto & Revistas en Q1 & \begin{tabular}{|l|} 
Scimag \\
0
\end{tabular} & 62 & NA & NA \\
\hline Índice de impacto & Revistas en Q2 & $\begin{array}{l}\text { Scimag } \\
0\end{array}$ & 63 & NA & NA \\
\hline Índice de impacto & Revistas en Q3 & \begin{tabular}{|l|} 
Scimag \\
0
\end{tabular} & 63 & NA & NA \\
\hline Índice de impacto & Revistas en Q4 & \begin{tabular}{|l|} 
Scimag \\
0
\end{tabular} & 57 & NA & NA \\
\hline Índice de impacto & Revistas en Q1 & JCR & 25 & NA & $\mathrm{NA}$ \\
\hline Índice de impacto & Revistas en Q2 & JCR & 26 & NA & NA \\
\hline Índice de impacto & Revistas en Q3 & JCR & 26 & NA & NA \\
\hline Índice de impacto & Revistas en Q4 & JCR & 26 & NA & NA \\
\hline
\end{tabular}

Fuente Elaboración Propia

\section{Conclusiones}

Coincide que los países que lideran los rankings mundiales con el mayor número de publicaciones en el área de finanzas sean los mismos que tengan más publicaciones en temas de finanzas sociales.

Con relación a los autores que más escriben sobre el tema en Latinoamérica, no es concluyente pues la mayoría solo tiene una sola publicación, por lo cual no hay algún autor que se destaque, al contrario, a nivel mundial hay varios referentes.

La cantidad de publicaciones en temas de finanzas sociales en nuestros países latinoamericanos es baja en comparación a las que se realizan en el mundo. Así mismo, a nivel global el tipo de publicaciones son artículos, cuando se compara con los países latinoamericanos se encuentra que sobresale la tesis de maestría.

Con respecto a los indicadores, el índice de impacto para Colombia es bajo en los tres años que ejemplo. Se aprecia un crecimiento a nivel mundial, pero en el país una reducción de las publicaciones.

${ }^{3}$ No es posible consultar el índice de impacto de Scimago y JCR del año 2019 y 2020 


\section{Referencias.}

LA Referencia. (1 de junio de 2020). LA Referencia. Obtenido de LA Referencia: http://www.lareferencia.info/vufind/Search/Results?lookfor=\%28finanzas+so ciales\%29+or+\%28inversi\%C3\%B3n+social\%29\&type=AllFields\&limit=20\&s ort=relevance

Nicholls, A., Paton, R., \& Emerson, J. (2015). Social Finance. Oxford University Press. doi:10.1093/acprof:oso/9780198703761.001.0001

Scimago Journal \& Country Rank. (31 de Mayo de 2020). Obtenido de Scimago Journal \& Country Rank: https://www.scimagojr.com/countryrank.php?category=2003\&area $=2000$

Scopus. (31 de mayo de 2020). Scopus. Obtenido de Elsevier: https://www-scopuscom.crai-

ustadigital.usantotomas.edu.co/term/analyzer.uri?sid=5d7e0b6d2aef725d98 7918da77e21a1c\&origin=resultslist\&src=s\&s=TITLE-ABS-

KEY\%28\%28social+finance\%29+or+\%28social+financial\%29+or+\%28+soci al+investment\%29\%29\&sort=plf-f\&sdt=b\&sot

Shiller, R. (2012). Las finanzas en una sociedad justa. Deusto. 